\section{Commentary: Flying solo without flying blind}

David L. Joyce, MD, MBA

"To become an ace a fighter must have extraordinary eyesight, strength, and agility, a huntsman's eye, coolness in a pinch, calculated recklessness, a full measure of courage and occasional luck!"

_James Doolittle

The parallels between air combat maneuvering and coronary artery bypass grafting extend well beyond the personality makeup and intestinal fortitude of the participants. The complexity of these procedures requires frequent practice, which is often difficult to acquire in a real-world environment, where life and death hang in the balance. Aviators have enjoyed a significant advantage over their cardiac surgical counterparts in that flight simulators offer a degree of realism that has remained elusive even in the best training models for the human heart. The tipping point that enabled this transition occurred on September 25, 1929, when Doolittle proved to the world that flying by instruments alone could be safely performed from takeoff to landing. Totally endoscopic coronary artery bypass notwithstanding, cardiac surgeons continue to depend on the advantages of exposure to complete a successful sortie when it comes to coronary revascularization. To this end, Saba and colleagues ${ }^{1}$ have introduced a simulation model that may offer unique advantages over existing approaches.

Efforts to simulate the technical complexities of coronary surgery originated with the development of a portable task station that featured silicon-based target vessels. Skills developed through the use of these low-fidelity models proved to be translatable to more-advanced assessments, such as operating on a beating-heart simulation. ${ }^{2}$ These benefits were expanded upon in the "Boot Camp" setting, where porcine models created an opportunity to further enhance the

\footnotetext{
From the Department of Surgery, Medical College of Wisconsin, Hub for Collaborative Medicine, Wauwatosa, Wis.

Disclosures: The author reported no conflicts of interest.

The Journal policy requires editors and reviewers to disclose conflicts of interest and to decline handling or reviewing manuscripts for which they may have a conflict of interest. The editors and reviewers of this article have no conflicts of interest.

Received for publication May 11, 2020; accepted for publication May 11, 2020; available ahead of print May 25, 2020.

Address for reprints: David L. Joyce, MD, MBA, Department of Surgery, Medical College of Wisconsin, Hub for Collaborative Medicine, $8701 \mathrm{~W}$ Watertown Plank Rd, Wauwatosa, WI 53226 (E-mail: djoyce@mcw.edu).

J Thorac Cardiovasc Surg 2021;161:e295-6

$0022-5223 / \$ 36.00$

Copyright (C) 2020 by The American Association for Thoracic Surgery

https://doi.org/10.1016/j.jtcvs.2020.05.034
}

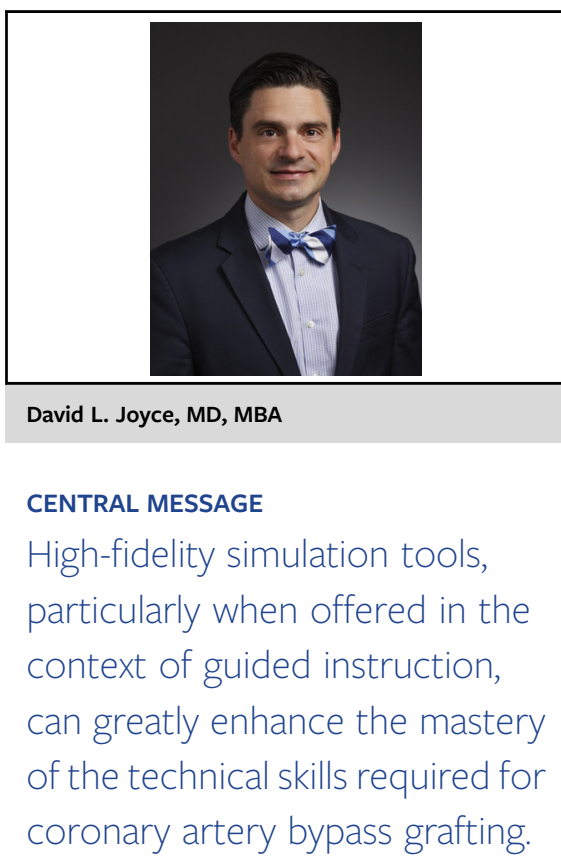

realism of anastomotic techniques. ${ }^{3}$ The introduction of computer-aided design and the use of 3-dimensional printing with hydrogel injection molding offer an opportunity for further progress toward the development of a high-fidelity coronary artery bypass grafting simulator.

Unlike the General Dynamics F-16 Fighting Falcon, which only has one seat for the pilot, the deployment of a cardiac surgical operating room requires the coordinated effort of multiple team members. ${ }^{4}$ Replicating this environment in a meaningful way requires a simulation experience more akin to the Red Flag exercises conducted at Nellis Air Force Base than the mere download of "Microsoft Flight." That said, the type of focused approach introduced here, which emphasizes deliberate practice of individual components, will undoubtedly enhance the value of more-complex training experiences.

Although the University of Rochester simulator overcomes the challenges of perishability and scalability associated with animal models, its value as a teaching tool requires an input that is often in short supply: "supervised instruction by experienced surgeons." Residents who have access to simulation in the presence of a dedicated mentor will achieve greater improvements than those who are relegated to flying blind. Installing a hydrogel heart model in the office of every faculty member may very well be the behavioral nudge that is needed to facilitate more teaching opportunities outside the operating room itself. 


\section{References}

1. Saba P, Melnyk R, Gosev I, Ghazi A, Hicks G. Development of a high-fidelity coronary artery bypass graft training platform using 3-dimensional printing and hydrogel molding. J Thorac Cardiovasc Surg. 2021;161:e291-3.

2. Fann JI, Caffarelli AD, Georgette G, Howard SK, Gaba DM, Youngblood P, et al. Improvement in coronary anastomosis with cardiac surgery simulation. J Thorac Cardiovasc Surg. 2008;136:1486-91.
3. Fann JI, Calhoon JH, Carpenter AJ, Merrill WH, Brown JW, Poston PS, et al. Simulation in coronary artery anastomosis early in cardiothoracic surgical residency training: the Boot Camp experience. J Thorac Cardiovasc Surg. 2010; 139:1275-81.

4. Joyce DL, Lahr BD, Maltais S, Said SM, Stulak JM, Nuttall GA, et al. Integration of simulation components enhances team training in cardiac surgery. $J$ Thorac Cardiovasc Surg. 2018;155:2518-24.e5.
See Article page e291.

\section{Commentary: Simulation - days of future past}

\author{
Frederick A. Tibayan, MD
}

My first coronary anastomoses were simulated with silicone vessels mounted on a wooden board-a portable task trainer known to many from the Boot Camp organized by the Thoracic Surgery Directors Association and the American Board of Thoracic Surgery. This and other models like it have been proven as robust and affordable methods of training that improve performance. ${ }^{1}$ One drawback of the "low-fidelity" simulation approach is relative lack of tactile feedback and anatomic/geometric realism. Cadaver and porcinebased "high-fidelity" simulations, in contrast, look and feel accurate but present significant financial and logistical hurdles. In this issue of the Journal, Saba and colleagues ${ }^{2}$ bridge the low- and high-fidelity approaches using a 3-dimensional printed coronary artery anastomosis model that can be dissected, cauterized, and sutured with a realistic feel.

This innovative approach is important because it is designed to mimic the geometry and mechanical properties achievable with biological models without the cost, biohazardous waste, and time constraints. Although only 5 trainees and 3 attendings were surveyed, the model was uniformly considered effective and realistic. As demonstrated in the supplementary video, anatomy can be customized to an individual patient's computed tomography scan, and models can be cheaply and quickly reproduced (after investment in a

From Division of Cardiothoracic Surgery, Department of Surgery, Oregon Health \& Science University, Portland, Ore.

Disclosures: The author reported no conflicts of interest.

The Journal policy requires editors and reviewers to disclose conflicts of interest and to decline handling or reviewing manuscripts for which they may have a conflict of interest. The editors and reviewers of this article have no conflicts of interest.

Received for publication June 1, 2020; revisions received June 1, 2020; accepted for publication June 2, 2020; available ahead of print June 26, 2020.

Address for reprints: Frederick A. Tibayan, MD, 3181 SW Sam Jackson Park Rd, Mail code: L353, Portland, OR 97239 (E-mail: tibayan@ohsu.edu).

J Thorac Cardiovasc Surg 2021;161:e296 $0022-5223 / \$ 36.00$

Copyright $₫ 2020$ Published by Elsevier Inc. on behalf of The American Association for Thoracic Surgery

https://doi.org/10.1016/j.jtcvs.2020.06.043
Check for updates

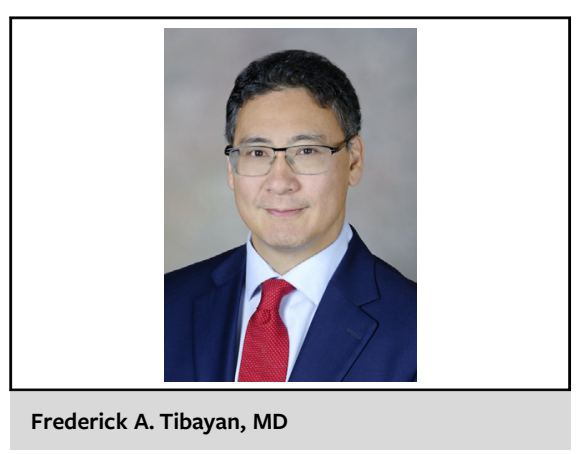

CENTRAL MESSAGE

3D-printed anastomosis model

bridges the gap between low-and

high-fidelity simulation, but chal-

lenges to implementation

remain.

3-dimensional printer). The novel technology developed by Saba and coauthors represents a step into the longvisualized future of cardiothoracic surgery simulation.

This step into the future can only be realized by addressing what we have learned in the past and are experiencing in the present. The rate-limiting and most important reagent in cardiothoracic simulation has and will continue to be dedicated faculty to instruct, design curricula, and give formative feedback. The tools we use in simulation improve with each passing year, but challenges still remain to incentivize teaching, fund resident education, and most importantly, identify faculty to mentor and train the next generation of surgeons.

\section{References}

1. Fann JI, Caffarelli AD, Georgette G, Howard SK, Gaba DM, Youngblood P, et al. Improvement in coronary anastomosis with cardiac surgery simulation. $J$ Thorac Cardiovasc Surg. 2008;136:1486-91.

2. Saba P, Ayers B, Melnyk R, Gosev I, Ghazi A, Hicks G. Development of a highfidelity coronary artery bypass graft training platform using 3-dimensional printing and hydrogel molding. J Thorac Cardiovasc Surg. 2021;161:e291-3. 